\title{
Images - Para-aortic lymphatic ectasia suggestive of testicular cancer retroperitoneal metastasis
}

\author{
Sonia Chahine, $\mathrm{PharmD}^{1}$; Paul Toren, $\mathrm{MD}, \mathrm{PhD}^{2}$ \\ ${ }^{1}$ Faculty of Medicine, Université Laval, Quebec City, QC, Canada; ${ }^{2}$ Division of Urology, Department of \\ Surgery, Faculty of Medicine, Université Laval, Quebec City, QC, Canada
}

Cite as: Can Urol Assoc J 2020 July 17; Epub ahead of print. http://dx.doi.org/10.5489/cuaj.6677

Published online July 17, 2020

$* * *$

\section{Introduction}

Para-aortic lymphatic ectasia is an anatomic variant which can be confused with metastatic lymph nodes. To date, there are few reports of this in the literature. It appears this variant is rare, but the true incidence is unknown. This case describes a patient with testicular cancer followed for unusual lesions initially suspected as retroperitoneal metastasis that were actually lymphatic ectasia. Through this case, we describe how this variant may be confused on imaging with retroperitoneal metastasis in the context of testicular cancer.

\section{Clinical history}

A 29-year-old male with no previous medical history presented with left testicular mass. Ultrasonography of the testis confirmed a $4.5 \times 4 \mathrm{~cm}$ mass highly suspicious. Tumor markers were normal (AFP:1.8, B-HCG $<1, \mathrm{LDH}: 166$ ). A left radical orchiectomy was performed, and the pathological examination diagnosed a mixed non-seminoma germ cell tumor (NSGCT) measuring $5 \times 4 \times 4 \mathrm{~cm}$ which was composed of $60 \%$ mature teratoma, $30 \%$ immature teratoma and $10 \%$ yolk sac tumor. The tumor was confined to the testis without evidence of lymphovascular invasion, stage pT1b.

A staging computed tomography scan was performed one month post-operatively which demonstrated left supra-hilar para-aortic lesions. They were described as low-density necrotic lymph nodes highly suspicious for metastasis. In particular, two nodes were described, the superior para-aortic measuring $18 \mathrm{~mm}$ in long axis, with an inferior paraaortic node measuring $11 \mathrm{~mm}$ in long axis (Fig. 1). No other suspicious retroperitoneal adenopathy was noted. 
Given their atypical necrotic appearance and the difficulty to access this location, percutaneous biopsy was undesirable, and it was decided with the patient to perform surveillance imaging with alternating magnetic resonance and computed tomography scans. A repeat CT scan 6 weeks later showed stability in size of the lesions in the left para-aortic region at the celiac trunk level.

The first magnetic resonance imaging was performed 2 months later showing similar left para-aortic and retrocrural adenopathy (Fig. 2). A second MRI 6 months later showed no changes. The third MRI performed 6 months later (16 months from an initial scan) showed that the lesions remained completely stable. At this point during the surveillance imaging, the diagnostic possibility of dilated lymphatic ectasia was raised by the radiologist, even though considered atypical in the left para-aortic position.

Six months later the diagnosis of para-aortic lymphatic ectasia was favored on a follow-up CT scan (Fig. 3), while the next CT scan, at 22 months from diagnosis described a bilobed nodularity of $36 \times 14 \mathrm{~mm}$ which was superimposable from the initial CT scan. A fourth MRI 28 months from diagnosis confirmed identical findings. Tumor markers and physical examination remained normal throughout all the surveillance process.

\section{Discussion}

Germ cell tumors of the testis have a typical pattern of metastatic dissemination via lymphatics. The teratoma found in this patient is a subtype of non-seminoma germ cell tumors (NSGCTs) identified in more than $47 \%$ of adults NSGCTs. The primary landing site of metastatic left-sided testis tumors is the left para-aortic area. Unlike the present case, these lesions are almost always initially below the left renal hilum. ${ }^{1}$ Because of the uncommon location of the lesions that were superior to the renal vessels and their unusual necrotic appearance, the decision to opt for observation was preferred to avoid unnecessary treatments. Aside from these lesions the patient had stage IA disease for which the relapse rate is low; hence the importance of a conservative approach. ${ }^{2}$

The presence of a cysterna chyli on abdominal CT scan is a rare finding but can be misinterpreted as an enlarged lymph node at first. The differentiating features of cysterna chyli on CT scans are a tubular structure, the absence of enhancement with intravenous contrast and low water attenuation. ${ }^{3}$ Few other structures can mimic lymphadenopathy such as an interrupted inferior vena cava with azygos continuation and other venous malformations. ${ }^{4,5}$

Abdominal lymphatic ectasia is rare finding which consists of the abnormal dilation of lymphatic vessels. In the pediatric population, lymphangiectasia is rarely encountered and appears mostly associated with underlying conditions. The most common locations of the lymphatic ectasia are the central conducting lymphatics and the pulmonary and intestinal lymphatic networks. ${ }^{6}$ In adults, it appears to be a congenital 
anatomical variant which affects the central conducting system. When the thoracic duct takes its origin higher in the abdomen, the lumbar lymphatic trunks join themselves at the level of L1-L2 and may be of greater diameter than normal. ${ }^{7}$ Other etiologies of lymphatic anomalies are lymphangioma, Gorham Stout disease and lymphangiomatosis. ${ }^{6}$ Previously, lymphangiography was the technology used to visualize the lymphatic system. ${ }^{8,9}$ Nowadays, high-resolution chest and abdominal CT with contrast agent can optimize demonstration of dilated lymphatic without invasive techniques. MRI studies with heavily T2-weighted sequences is also commonly used to delimit the central conducting lymphatics. ${ }^{6,10}$

A review of the literature revealed one other case where lymphatic ectasia was initially misdiagnosed as abnormal abdominal lymphadenopathy. It involves the case of a 34-year-old treated for Hodgkin's disease for whom dilated lumber trunks were misinterpreted as residual abnormal lymphadenopathy in the para-aortic region on a CT scan. In this case, both lymphangiography and MRI confirmed the presence of retroperitoneal dilated lymphatic vessels. ${ }^{7}$ Enlarged para-aortic lymphatic ducts was also described in a series of 3 cases, where the suspected etiology was previous abdominal surgery. ${ }^{9}$ Ligation or iatrogenic disruption of the thoracic duct or high abdominal lymphatic vessels could cause dilation of lower lymphatic structures. In our case, an anatomical variant of the thoracic duct appears the most plausible etiology since our patient was otherwise healthy and young with no past medical history.

\section{Conclusions}

This case demonstrates how a lymphatic anatomic variant of the lumbar lymphatic ducts may appear as metastasis on imaging. It highlights the worth of considering this variant in the differential diagnosis of retroperitoneal lymphadenopathy and the value of a conservative approach in testicular cancer patients with unusual lesions in the retroperitoneum. 


\section{References}

1. Stephenson AJ, Gilligan TD. Neoplasms of the Testis. In: Wein AJ, Kavoussi LR, Partin AW, Peters CA, editors. Cambell-Walsh Urology 1. 11th ed. Philadelphia, PA: Elsevier; 2015. p. 784-814.

2. Aydin AM, Zemp L, Cheriyan SK, et al. Contemporary management of early stage testicular seminoma. Transl Androl Urol 2020;9(Suppl 1):S36-s44.

3. Smith TR, Grigoropoulos J. The cisterna chyli: incidence and characteristics on CT. Clin Imaging 2002;26(1):18-22.

4. Lien $\mathrm{HH}$, Talle K. Normal and anomalous structures simulating retroperitoneal lymphadenopathy at computed tomography. Acta Radiol 1988;29(4):385-90.

5. Yilmaz U, Halilcolar H, Unsal I, et al. Isolated interrupted inferior vena cava with azygos continuation mimicking paraesophageal lymph node enlargement. Monaldi Arch Chest Dis 2006;65(4):228-30.

6. Malone LJ, Fenton LZ, Weinman JP, et al. Pediatric lymphangiectasia: an imaging spectrum. Pediatric Radiology 2015;45(4):562-69.

7. Williams MP, Olliff JFC. Case report: Computed tomography and magnetic resonance imaging of dilated lumbar lymphatic trunks. Clinical Radiology 1989;40(3):321-22.

8. Chavhan GB, Amaral JG, Temple M, et al. MR Lymphangiography in Children: Technique and Potential Applications. RadioGraphics 2017;37(6):1775-90.

9. Verbanck JJ, Vermeulen JT, Rutgeerts LJ, et al. Dilated abdominal paraaortic lymphatic duct: a possible pitfall in retroperitoneal US. Radiology 1988;167(3):701-2.

10. Govil S, Justus A, Lakshminarayanan R, et al. Retroperitoneal lymphatics on CT and MR. Abdom Imaging 2007;32(1):53-55.

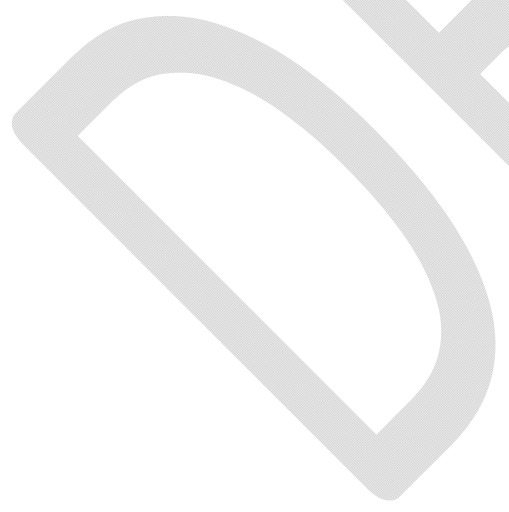




\section{Figures and Tables}

Fig. 1A. Coronal and axial staging computed tomography performed one-month postorchiectomy shows left supra-hilar para-aortic lesions described as low-density necrotic lymph nodes highly suspicious for metastasis. The superior para-aortic measuring $18 \mathrm{~mm}$ in long axis, with an inferior para-aortic node measuring $11 \mathrm{~mm}$ in long axis.

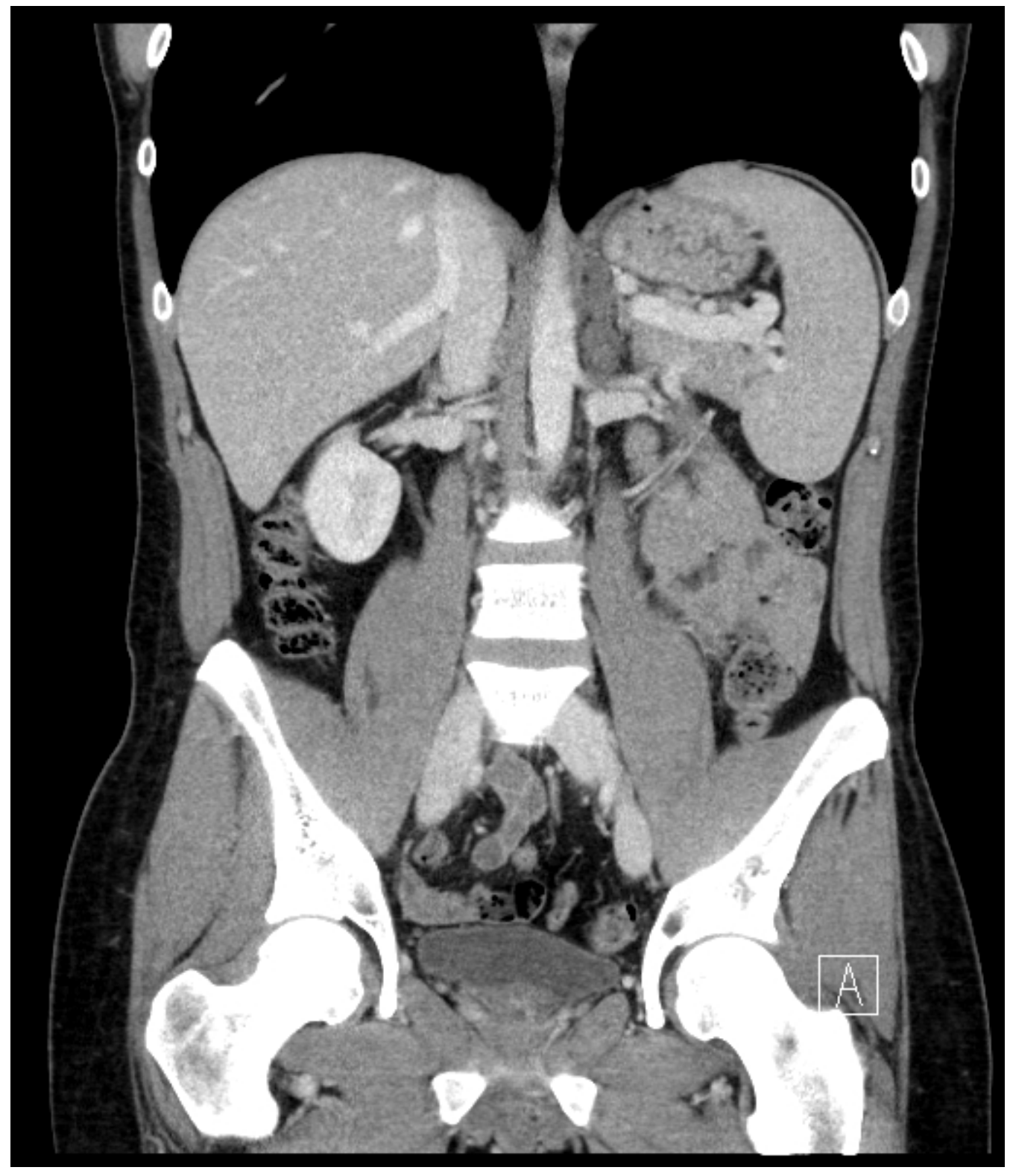


Fig. $1 B$.

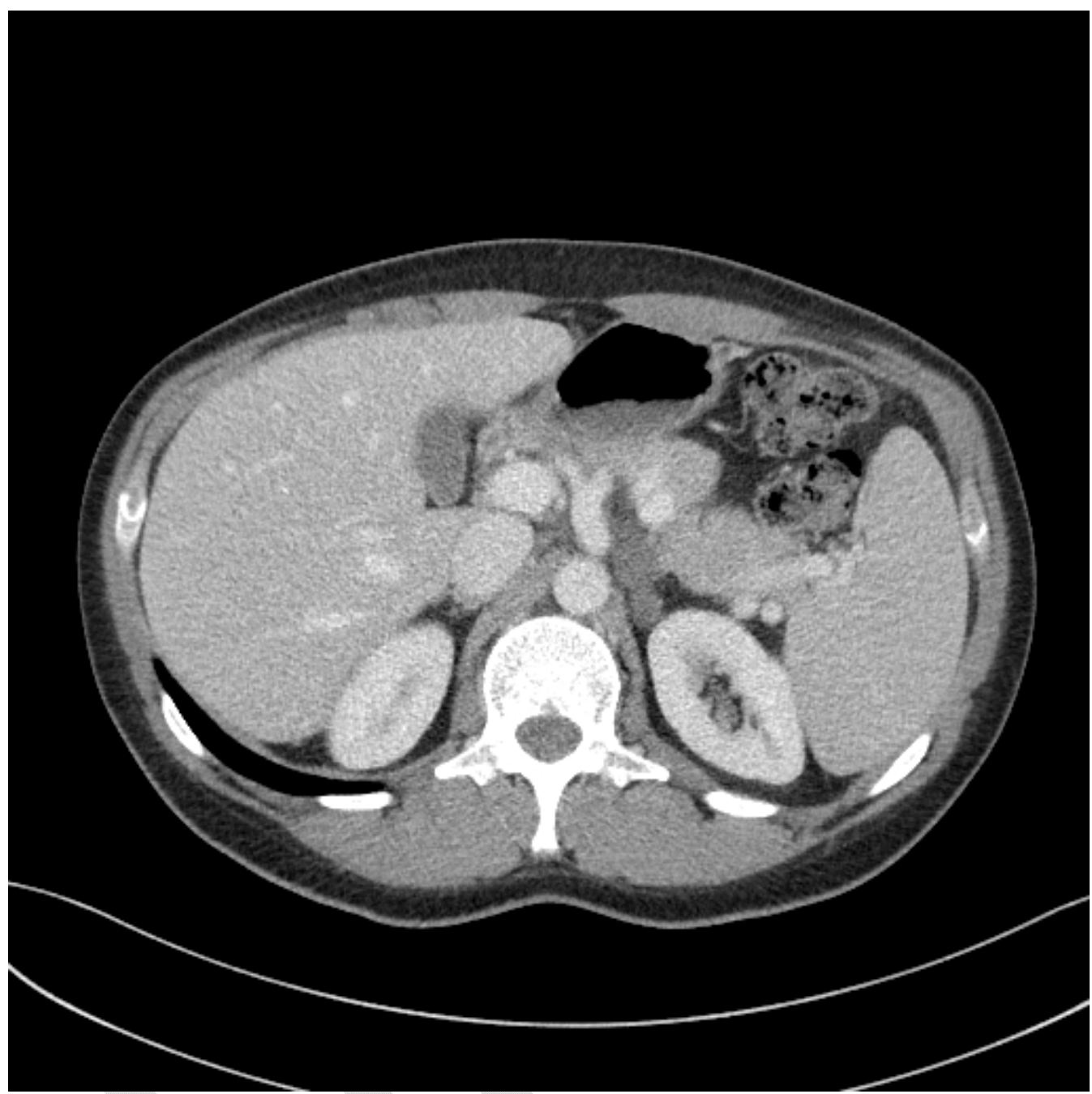


Fig. 2. First magnetic resonance imaging performed 2 months later showing similar left para-aortic and retrocrural adenopathy.

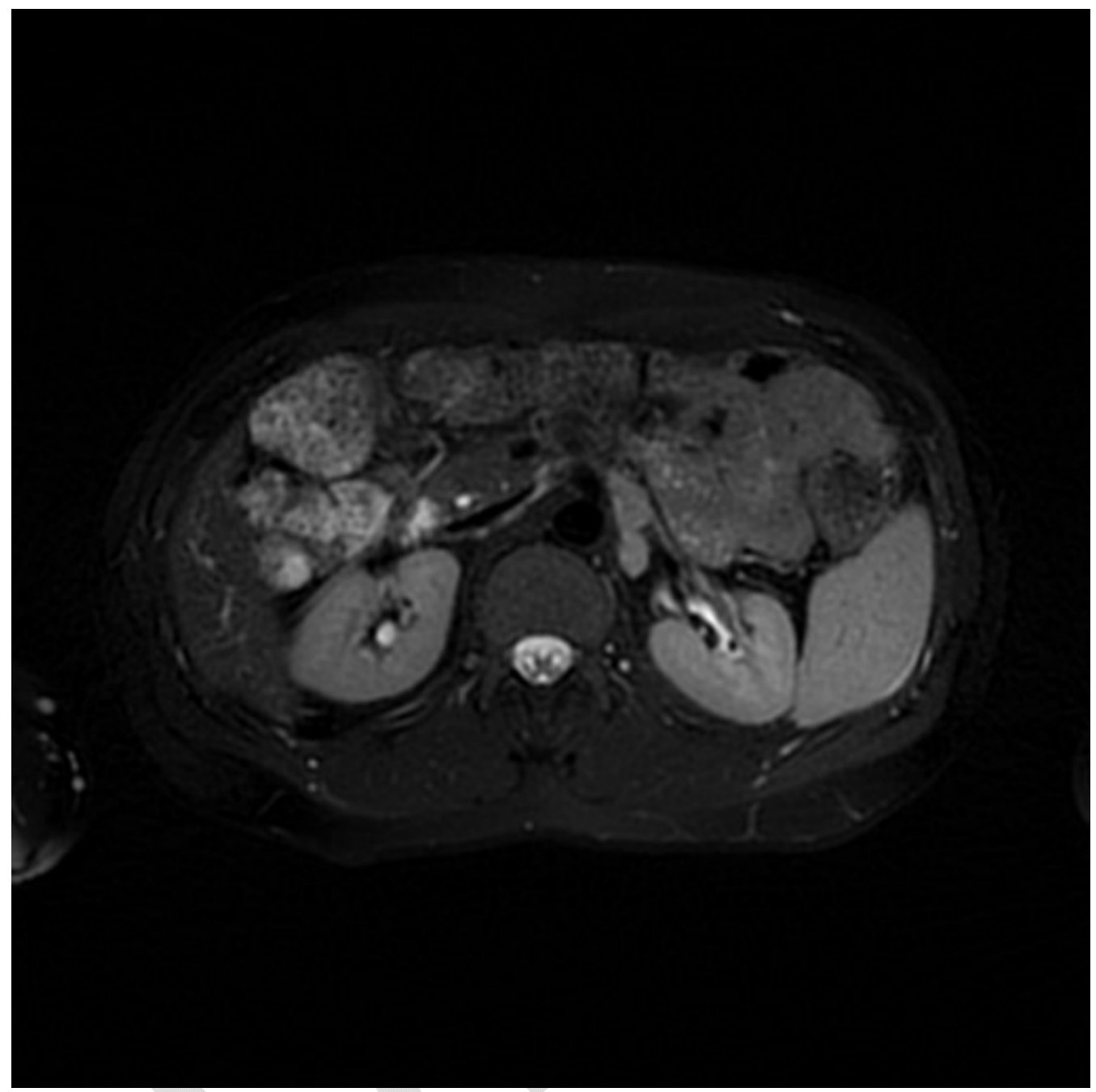


Fig. 3. Third followup computed tomography scan.

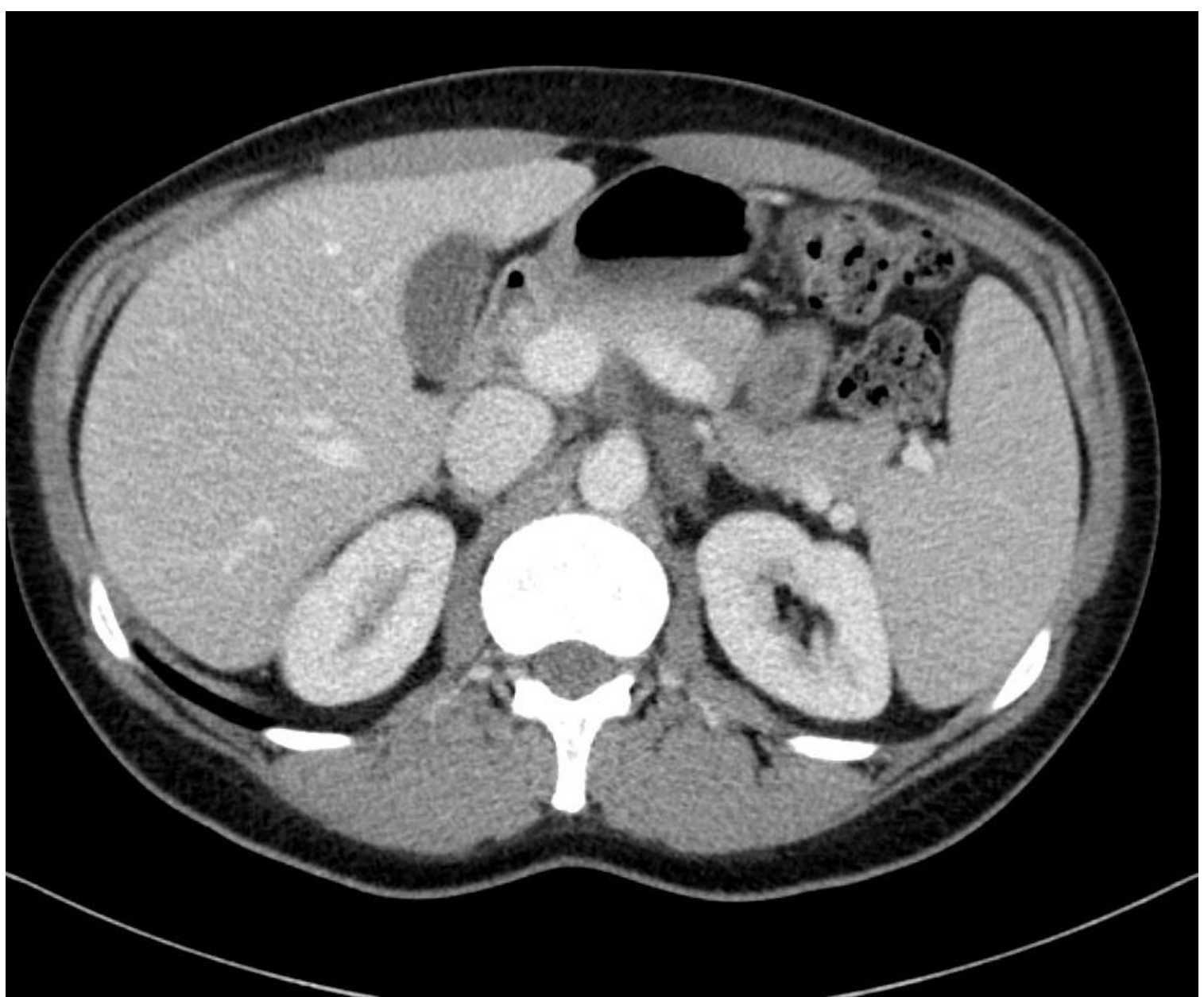

